\title{
Is less always better? Keyhole and standard subtemporal approaches: evaluation of temporal lobe retraction and surgical volume with and without zygomatic osteotomy in a cadaveric model
}

\author{
Serdar Ercan, MD, ${ }^{1}$ Alba Scerrati, MD, ${ }^{2}$ Phengfei Wu, MD, PhD, ${ }^{3}$ Jun Zhang, $\mathrm{PhD},{ }^{4}$ and \\ Mario Ammirati, MD, MBA ${ }^{1}$ \\ 'Dardinger Skull Base Laboratory, Department of Neurological Surgery, Ohio State University Wexner Medical Center, \\ Columbus, Ohio; ${ }^{2}$ Catholic University, Institute of Neurosurgery, Rome, Italy; ${ }^{3}$ The First Affiliated Hospital of China Medical \\ University, Department of Neurosurgery, Shenyang, Lianoning, China; and ${ }^{4}$ Department of Radiology, Ohio State University, \\ Columbus, Ohio
}

\begin{abstract}
OBJECTIVE The subtemporal approach is one of the surgical routes used to reach the interpeduncular fossa. Keyhole subtemporal approaches and zygomatic arch osteotomy have been proposed in an effort to decrease the amount of temporal lobe retraction. However, the effects of these modified subtemporal approaches on temporal lobe retraction have never been objectively validated.

METHODS A keyhole and a classic subtemporal craniotomy were executed in 4 fresh-frozen silicone-injected cadaver heads. The target was defined as the area bordered by the superior cerebellar artery, the anterior clinoid process, supraclinoid internal carotid artery, and the posterior cerebral artery. Once the target was fully visualized, the authors evaluated the amount of temporal lobe retraction by measuring the distance between the base of the middle fossa and the temporal lobe. In addition, the volume of the surgical and anatomical corridors was assessed as well as the surgical maneuverability using navigation and 3D moldings. The same evaluation was conducted after a zygomatic osteotomy was added to the two approaches.
\end{abstract}

RESULTS Temporal lobe retraction was the same in the two approaches evaluated while the surgical corridor and the maneuverability were all greater in the classic subtemporal approach.

CONCLUSIONS The zygomatic arch osteotomy facilitates the maneuverability and the surgical volume in both approaches, but the temporal lobe retraction benefit is confined to the lateral part of the middle fossa skull base and does not result in the retraction necessary to expose the selected target.

https://thejns.org/doi/abs/10.3171/2016.6.JNS16663

KEY WORDS keyhole; subtemporal; temporal lobe retraction; surgical technique

$\mathrm{V}$ ISUALIZATION of the central skull base is challenging. The subtemporal approach is one of the routes used to expose the interpeduncular fossa and the basal diencephalon. A certain degree of temporal lobe retraction is needed to gain sufficient surgical exposure..$^{2,3}$, ${ }^{11,30}$ Keyhole subtemporal approaches and a zygomatic arch osteotomy have been proposed in an effort to decrease the amount of temporal lobe retraction. $3,4,6,7,10,13,15,16,18,24$ However, the effects of these modified subtemporal approaches on temporal lobe retraction have never been objectively validated. The purpose of our research was to compare the temporal lobe retraction and surgical/anatomical corridors of the keyhole and classic subtemporal approaches.

\section{Methods}

Specimens and Equipment

We used 4 fresh-frozen silicone-injected cadaver heads.

ABBREVIATIONS PCA = posterior cerebral artery; SCA = superior cerebellar artery.

SUBMITTED March 14, 2016. ACCEPTED June 8, 2016.

INCLUDE WHEN CITING Published online September 16, 2016; DOI: 10.3171/2016.6.JNS16663. 

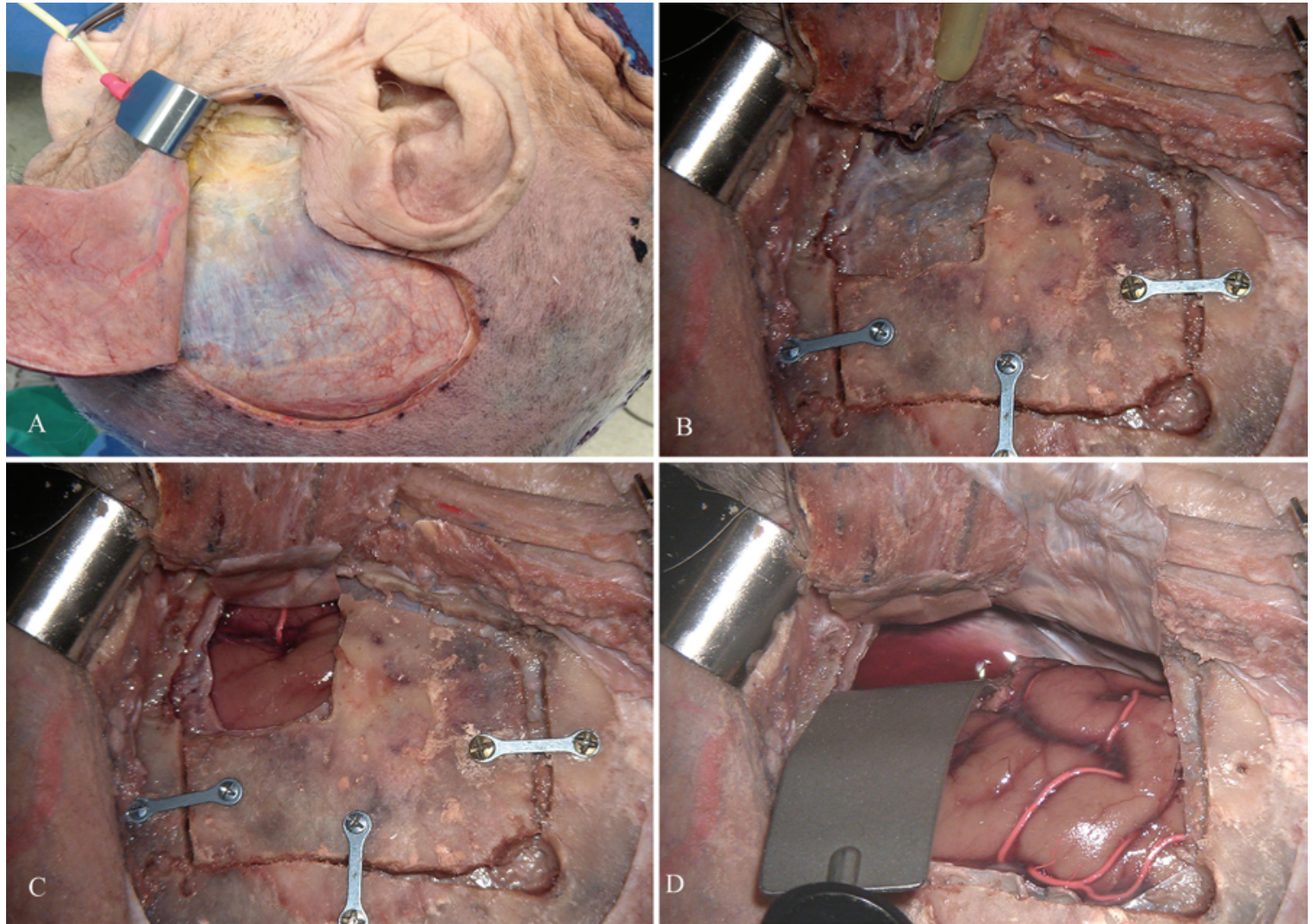

FIG. 1. Photographs of the surgical procedure. A: The question mark-shaped skin incision is shown. B: A $2.5 \times 2.5-\mathrm{cm}$ keyhole craniotomy was performed. After the classic craniotomy was completed, the bone flap was reattached rigidly with microplates.

C and D: The temporal dura was incised just at the edge of the keyhole craniotomy (C) and classic craniotomy (D) separately. The temporal lobe was retracted using a self-retaining retractor (D). Figure is available in color online only.

All specimens were stored in a $66 \%$ ethyl alcohol solution to minimize hardening of the brain. High-resolution CT scans were obtained for every specimen, with the following parameters: slice thickness $1 \mathrm{~mm}$, contiguous nonoverlapping slices, gantry setting $0^{\circ}$, scan window diameter $225 \mathrm{~mm}$, pixel size $>0.44 \times 0.44 . .^{22}$ Each head was rigidly fixed in a Mayfield head holder in a position simulating the actual surgical approach and registered on an image guidance workstation with a 6-marker digitizing probe (Stryker Instruments). We used an FS Moller-Wedel International FS 4-20 microscope.

\section{Surgical Approaches}

The surgical approaches were performed using standard microsurgical instruments and a surgical microscope with a magnification range of $\times 3$ to $\times 25$. A highspeed drill was used when indicated. Touching the tip of the digitizing probe to the points of interest while the probe was in view of the camera created an anatomical data set of the selected points. A computer connected to the Stryker system stored data files in the form of $\mathrm{x}, \mathrm{y}$, and $\mathrm{z}$ coordinates (in $\mathrm{mm}$ ) of each point, and the accompanying software calculated the distances between any two points of interest.

On each side of the cadaver head we performed the keyhole and the subtemporal approaches, giving us 4 pairs of approaches, each pair consisting of keyhole and sub- temporal approaches performed on the same side on the same cadaver (Fig. 1). These pairs formed the basis of our anatomical and surgical space evaluation, which is therefore a comparative evaluation.

\section{Position}

The cadaver heads were placed in the lateral position, with the long axis of the head parallel to the floor and held in place with a Mayfield head holder to simulate the standard surgical position. The dissection was performed with standard microsurgical instruments, power drills, and a surgical microscope.

\section{Incision}

The question mark-shaped skin incision was made, beginning at the level of the lower edge of the zygomatic arch, $1 \mathrm{~cm}$ anterior to the tragus, and extending to the superior temporal line. The scalp flap was reflected anteriorly. The temporal muscle was separated from the skull and dissected downward to be held in place at the upper edge of the zygomatic arch.

\section{Craniotomy for the Keyhole Approach}

A bur hole was placed above the temporomandibular joint, and a rectangular-shaped craniotomy $(2.5 \mathrm{~cm}$ in width and $2.5 \mathrm{~cm}$ in height) was executed close to the 


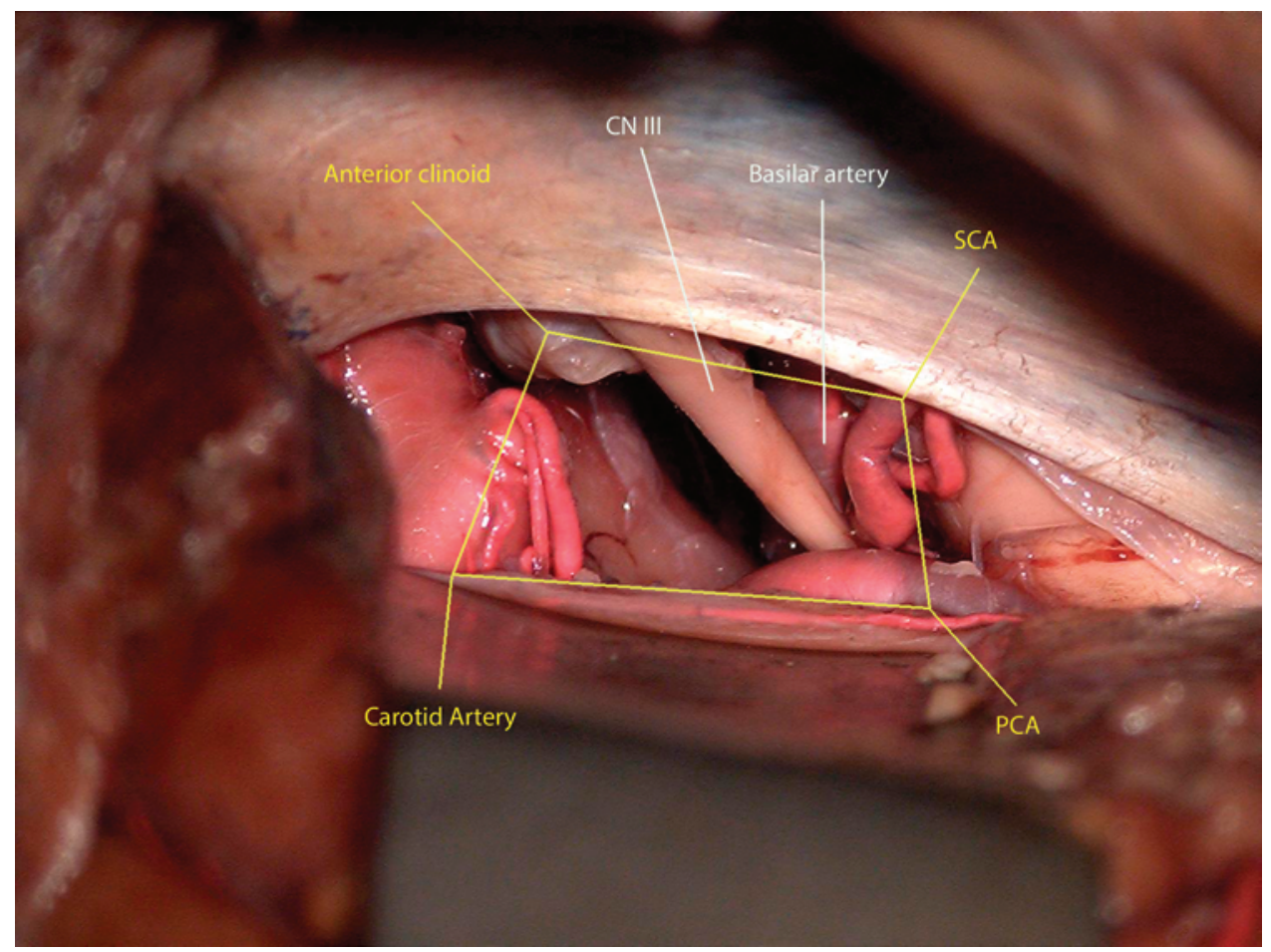

FIG. 2. The target area is shown, which is limited by the anterior clinoid process, SCA, PCA, and carotid artery. CN III = cranial nerve III. Figure is available in color online only.

cranial base using the craniotome (Medtronic Midas Rex Legend EHS). ${ }^{18,28,29,32}$

\section{Craniotomy for the Classic Approach}

The keyhole craniotomy was extended superiorly and posteriorly up to the squamosal suture. Thus, the temporal craniotomy was performed two-thirds anterior to the external acoustic canal using a high-speed drill and a craniotome. The squamous temporal bone was drilled down to the floor of the middle fossa. ${ }^{23}$ After the classic craniotomy was completed, the bone flap was reattached with microplates (Codman/Johnson \& Johnson), leaving in place only the opening of the keyhole craniotomy. Performing the craniotomies in this order made measurements of the two approaches easier to execute.

\section{Section of the Zygomatic Arch}

The zygomatic arch was sectioned with two vertical cuts: a posterior cut before the temporomandibular joint, and an anterior cut behind the union of the zygomatic arch and the zygomatic bone. Afterward the zygomatic arch was moved downward with the masseter muscle. The new temporal bone area, which was exposed after the section of the zygomatic arch, was drilled with a high-speed drill, thus extending the areas of the previous two craniotomies.

\section{Dural Incision}

The temporal dura was incised and inferiorly retracted.

\section{Evaluation of Temporal Lobe Retraction}

After dural opening, the temporal lobe was retracted using a Budde Halo retractor system (Integra), with a 10-mm-tip spatula.

\section{Target Area}

A rectangular target area was defined by connecting these 4 points: 1) the point where the superior cerebellar artery (SCA) crosses the free edge of the tentorium (posterolateral); 2) the point where the anterior clinoid process crosses the free edge of the tentorium (anterolateral); 3) the most distal point of the exposed supraclinoid internal carotid artery (anteromedial); and 4) the most distal point of the exposed posterior cerebral artery (PCA, posteromedial; Fig. 2).

A navigation probe was used to determine the cartesian coordinates $(\mathrm{x}, \mathrm{y}, \mathrm{z})$ of the selected points. The target area was calculated using the formula:

$$
1 / 2\left|\begin{array}{ccc}
1 & 1 & 1 \\
r-k & s-m & t-n \\
u-k & v-m & w-n
\end{array}\right|
$$

in reference to a triangle in 3D space that has the following coordinates: $(\mathrm{k}, \mathrm{m}, \mathrm{n}),(\mathrm{r}, \mathrm{s}, \mathrm{t})$, and $(\mathrm{u}, \mathrm{v}, \mathrm{w})$. The target areas of all cadavers were approximately the same (mean $17.1 \pm 1.16 \mathrm{~mm}^{2}$ ).

\section{Evaluation of Temporal Lobe Retraction}

The retractor position was adjusted so as to fully visualize the target area. Once the target area has visualized the position of the retractor, it remained constant throughout the remainder of the dissection and measurements. ${ }^{28}$ The temporal lobe retraction was defined as the distance 


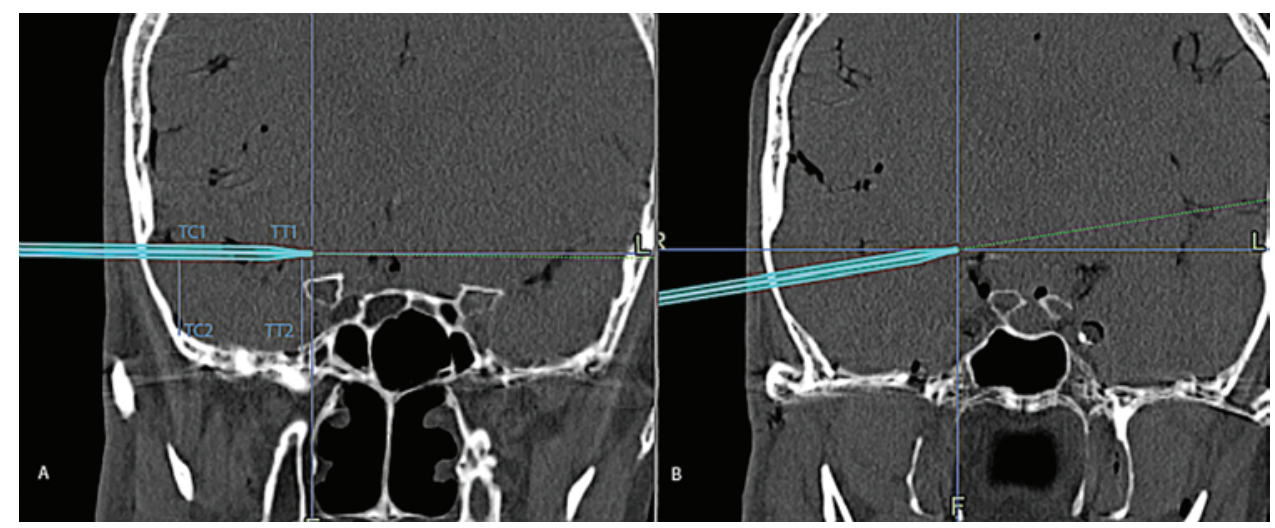

FIG. 3. There were not any significant differences in the medial retraction distance between the approaches. A: Temporal lobe retraction. B: Temporal lobe retraction after zygomatic arch osteotomy. TC1 = superior point of the retractor at the edge of the craniotomy; TC2 = vertical projection of the TC1 at the skull base beside temporal bone; TT1 = superior point of the retractor at the middle part of the target; TT2 = free edge of the tentorium close to the target. Figure is available in color online only.

between the retractor blade and the skull base. We calculated two different distances, one at the lateral-most part of the retractor blade and one at the medial-most part of the retractor blade. The lateral distance is the vertical distance between the middle of the retractor at the edge of the craniotomy (TC1) and the skull base at the same level (TC2); this distance (TC1 to TC2) is the retraction of the superficial temporal lobe. The medial distance is the vertical distance between the middle of the retractor at the level of the target (TT1) and the free edge of the tentorium close to the target (TT2); this distance (TT1 to TT2) is the retraction of the deep temporal lobe (Fig. 3). All cartesian points were determined using the cranial navigation system (Stryker), and the distance was calculated using the following formula: $d=\sqrt{ }\left(x_{2}-x_{1}\right)^{2}+\left(y_{2}-y_{1}\right)^{2}+\left(z_{2}-z_{1}\right)^{2}$.

We performed retraction measurements first on the keyhole approach. Afterward the temporal bone flap, which was previously reattached, was removed, and the same process was repeated for determination of the retraction for the classic craniotomy.

\section{Volume of the Corridors}

Two different corridors were identified after the craniotomies and temporal lobe retraction: the anatomical corridor and the surgical corridor.

\section{Volume of the Anatomical Corridor (Space)}

We define the anatomical corridor as the volume of the space that exists after temporal lobe retraction. This volume was calculated as follows: the surface of the surgical area was covered by wet cotton fabric and then filled with a mixture prepared by adding $6 \mathrm{ml}$ of water to $4 \mathrm{ml}$ of resin (ST-504, Strata-Tech Inc.). Ten minutes later, the mixture solidified, resulting in a resin mold of the anatomical corridor that could be easily removed from the surface of the exposed brain. This mold was then submerged into a cubic unit-scaled box, and the volume difference after submersion compared with presubmersion represented the volume of the mold, and hence the volume of the anatomical corridor.

\section{Volume of the Surgical Corridor}

We defined the surgical corridor as the corridor/volume in which surgical maneuvers on the target area can be performed. The surgical corridor (space) is part of the anatomical space; indeed, it represents that part of the anatomical space where surgical maneuvers can be performed. The corridor is defined as a frustum whose base is the area of the target, which is constant for all the approaches, and whose top is the area of the craniotomy. The height of the frustum is the distance between the craniotomy and the target. The volume of the surgical corridor was calculated by cartesian points selected at the base and at the top of the frustum using 2 different techniques: 1) classic mathematical formula, in which the volume of the pyramid frustum was determined by the following formula: $V=H / 3\left(S_{b 1}+S_{b 2}+\sqrt{ } S_{b 1} S_{b 2}\right)$, in which $S_{b 1}$ and $S_{b 2}$ are the base and topside lengths of the truncated pyramid and $\mathrm{H}$ is the height; and 2) the 3D model of the surgical corridor was mapped using Blender software (Blender 2.75), and the volume and the angles between the target and the corridor were determined using the same software. In each pair of approaches the ratio between the volume of the anatomical and surgical volume was used to compare the approaches.

\section{Angles of Attack}

Angles of attack were calculated using cartesian points at the navigation system (Stryker) and the 3D mapping program (Blender). The vertical angle of attack was a hypothetical line from the inferior part of the craniotomy to the superior part of the target (SL, superior line), and from the superior part of the craniotomy to the inferior part of the target (IL, inferior line), depicting the maximum angle of attack to the superior and inferior parts of the target. The angle between these two lines (SL and IL) was accepted as the maximum vertical angle of attack to the target. The horizontal angle of attack was a hypothetical line from the anterior part of the craniotomy to the posterior part of the target (PL, posterior line), and from the posterior part of the craniotomy to the anterior part of the target (AL, anterior line), illustrating the maximum angle of at- 
TABLE 1. Comparisons of the approaches

\begin{tabular}{lllll}
\hline \multicolumn{1}{c}{ Variable } & \multicolumn{1}{c}{$\mathrm{K}$} & \multicolumn{1}{c}{$\mathrm{C}$} & $\mathrm{K}+\mathrm{Z}$ & $\mathrm{C}+\mathrm{Z}$ \\
\hline Temporal lobe retraction $(\mathrm{mm})$ & & & & \\
\hline$\quad$ Lateral & 21.86 & 21.94 & 17.11 & 16.43 \\
\hline Medial & 26.75 & 27.19 & 26.16 & 26.90 \\
\hline Anatomical corridor $\left(\mathrm{cm}^{3}\right)$ & 33 & 32 & 33 & 31 \\
\hline Surgical corridor $\left(\mathrm{cm}^{3}\right)$ & 15.87 & 29.63 & 20.26 & 34.12 \\
\hline Vertical angle $\left(^{\circ}\right)$ & 45 & 89 & 68 & 111 \\
\hline Horizontal angle $\left({ }^{\circ}\right)$ & 59 & 103 & 59 & 103 \\
\hline
\end{tabular}

$\mathrm{C}=$ classic craniotomy $\mathrm{C}+\mathrm{Z}=$ classic craniotomy with zygomatic arch osteotomy; $\mathrm{K}=$ keyhole craniotomy; $\mathrm{K}+\mathrm{Z}$ = keyhole craniotomy with zygomatic arch osteotomy.

All values given are means.

tack to the anterior and posterior parts of the target. The angle between these two lines (AL and PL) was accepted as the maximum horizontal angle of attack to the target.

We used the following procedures to calculate the angles identified by the intersection of the 2 lines. All cartesian coordinates $(\mathrm{x}, \mathrm{y}$, and $\mathrm{z}$ ) were converted into polar coordinates, which is a coordinate system for $3 \mathrm{D}$ space in which the position of a point is specified by 3 numbers: the radial distance $(R)$, polar angle $(\theta)$, and azimuth angle $(\varphi)$. The polar angle and the azimuth angle are used for calculating the vertical angle of attack and the horizontal angle of attack, respectively. The formulas used are as follows: vertical angle $=180-\left(\theta_{\mathrm{s}}+\theta_{\mathrm{i}}\right)$, and horizontal angle $=180-$ $\left(\varphi_{\mathrm{a}}+\varphi_{\mathrm{p}}\right)$, in which $\mathrm{s}=$ superior, $\mathrm{i}=$ inferior, $\mathrm{a}=$ anterior, and $\mathrm{p}=$ posterior.

\section{Statistical Analysis}

Using SPSS software (version 16.0, SPSS Inc.), results were compared using a paired t-test.

\section{Results}

\section{Retraction Distance}

The mean retraction distance between the temporal lobe and the base of the temporal bone at the lateral temporal lobe region was similar for the two approaches (mean distance $21.86 \mathrm{~mm}$ and $21.94 \mathrm{~mm}$, respectively, for the keyhole and the classic approaches; $p=0.801$ ). The medial retraction distance was also similar in the two approaches (mean $26.75 \mathrm{~mm}$ and $27.19 \mathrm{~mm}$, respectively, for the keyhole and the classic approaches; $p=0.087$ ). Removal of the zygomatic arch enabled a more inferior-superior visual angle to the target. Due to this angle, the retraction at the lateral region (TC1 to TC2) was significantly reduced for both the keyhole and the classic craniotomy (mean 17.11 vs $16.43 \mathrm{~mm}$, respectively, for the keyhole and classic approaches; $\mathrm{p}<0.001$ ), but there were not any significant differences between the keyhole and classic approaches after the zygomatic arch osteotomy $(p=0.175)$. However, the mean retraction distance near the target (TT1 to TT2) was not affected by this osteotomy (mean distance $26.16 \mathrm{~mm}$ and $26.90 \mathrm{~mm}$, respectively, for the keyhole and classic approaches; p = 0.350; Table 1, Figs. 3 and 4).

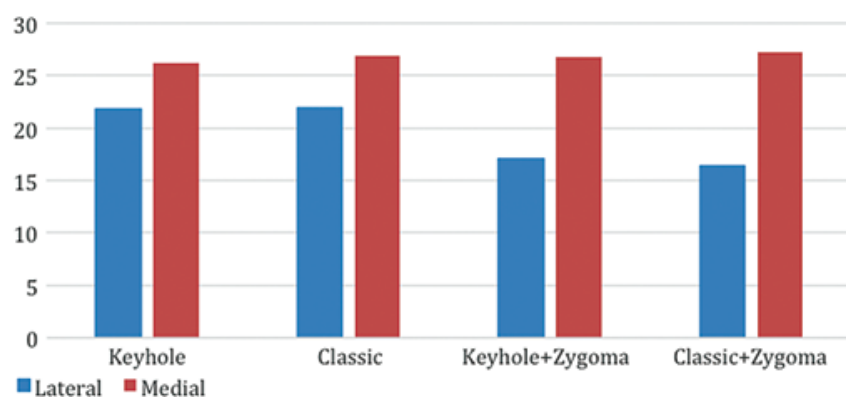

FIG. 4. Bar graph illustrating the distance of temporal lobe retraction $(\mathrm{mm})$ at the lateral and medial temporal lobe in different approaches. Figure is available in color online only.

\section{Volume of the Anatomical Corridor}

The mean volume of resin mold was similar for all craniotomies (mean $33.75 \mathrm{ml}$ ). The retractor lifted the temporal lobe not only at the site where it was placed, but also anteriorly and posteriorly (Fig. 5).

\section{Volume of the Surgical Corridor}

The mean volume of the surgical corridor was 15.875 $\mathrm{cm}^{3}$ for the keyhole craniotomy; this volume represented a mean of $47 \%$ of the anatomical corridor. The mean volume of the surgical corridor was $29.63 \mathrm{~cm}^{3}$ for the classic craniotomy, which represented a mean of $87 \%$ of the anatomical corridor. The volume of the surgical corridor associated with the classic approach was significantly larger than that associated with the keyhole approach $(\mathrm{p}$ $=0.026$ ). Zygomatic arch removal provided an extra 4.39 $\mathrm{ml}$ of surgical volume to both the keyhole and the classic craniotomy (Fig. 6). With the temporal lobe retracted, the temporal basal veins are stretched; these stretched veins are visualized better with the classic craniotomy than with the keyhole craniotomy, where the endoscope may help in this visualization.

\section{Angle of Attack}

The maximum angle was $45^{\circ}$ for the vertical and $59^{\circ}$ for the horizontal angles of attack for the keyhole approach. The angles were significantly wider for the classic craniotomy $\left(89^{\circ}\right.$ and $103^{\circ}$, respectively, for the vertical and horizontal angles; $\mathrm{p}<0.001)$. There were no statistically significant differences in the horizontal attack after the removal of the zygomatic arch for both the keyhole and the classic craniotomy $(p=0.796)$. In contrast, removal of the zygomatic arch provided approximately $22^{\circ}$ extra vertical attack angle for both approaches, which was significantly greater than pre-osteotomy; $\mathrm{p}<0.001$; Fig. 7).

\section{Discussion}

Extensive temporal lobe retraction can result in postoperative seizures, aphasia, venous complications, or other focal neurological deficits. ${ }^{2,11,30}$ The stretch of the inferior anastomotic vein (vein of Labbé) and of other temporal lobe basal veins are potentially serious complications that can lead to massive infarction and even death in some cases. ${ }^{5,14,19,31}$ Keyhole and endoscopic subtemporal ap- 

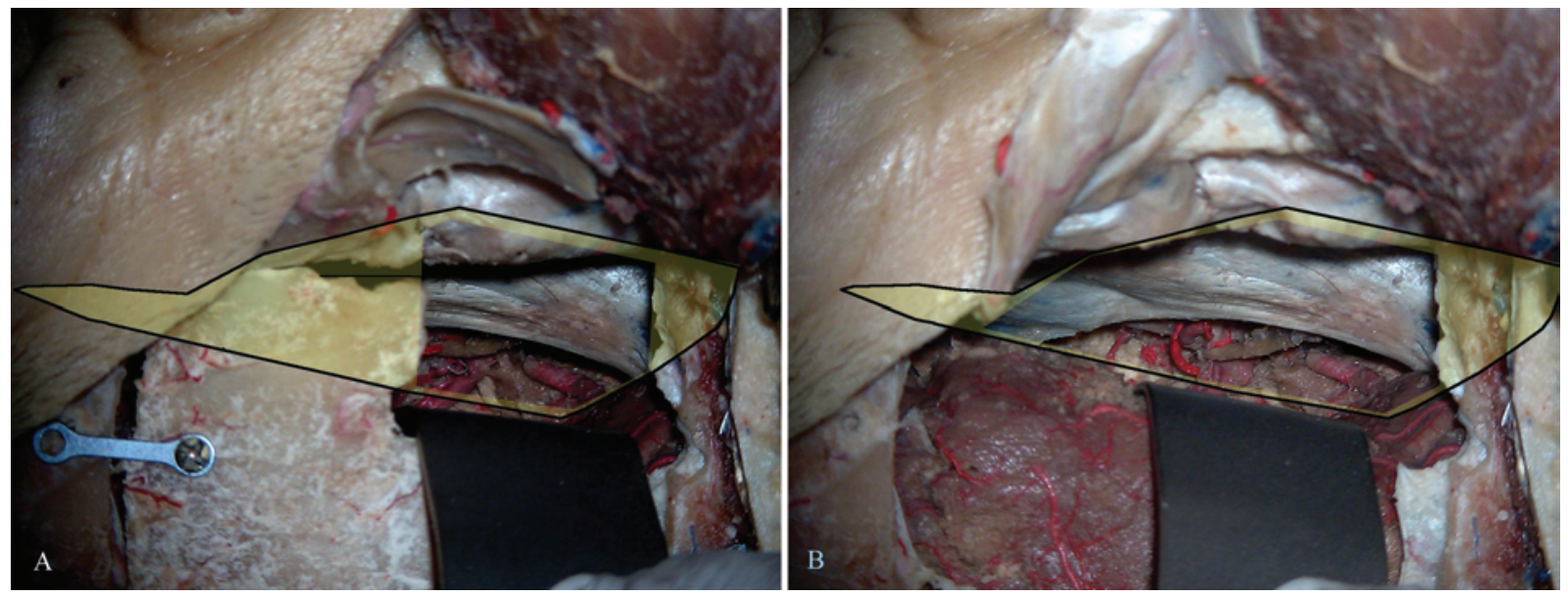

FIG. 5. The retractor lifts the temporal lobe not only at the retraction site, but also anteriorly and posteriorly. The area encircled by the black line represents the anatomical volume while the part that is not shaded in yellow represents the surgical volume. It is evident that the surgical volume of the keyhole craniotomy $(\mathbf{A})$ is less than that of the classic craniotomy $(\mathbf{B})$. Figure is available in color online only.

proaches, as well as zygomatic arch osteotomy, have been proposed to reduce these complications. ${ }^{3,15,17,18}$ The term "keyhole surgery" does not refer to a specific technique or approach but rather to a philosophy that emphasizes using the smallest opening necessary to achieve the anatomical goals of surgery.9,21,25 Ideally, the aim of any neurosurgical procedure used to access deep brain lesions should be minimal brain retraction. Wang, Zhu, and Taniguchi stated that in the subtemporal approach, brain retraction could be reduced by performing keyhole craniotomies. ${ }^{26,28,29,31}$ Taniguchi et al. also claim that the concomitant injury to the bridging veins (including the vein of Labbé)-which are mentioned as frequent complications, especially in cases in which wide exposure or deep view was required-can be prevented by the keyhole craniotomy. ${ }^{26}$ Indeed, keyhole principles do not always facilitate an approach to deep targets because of difficult brain retraction and minimal ability to perform needed neurovascular manipulations. Fatemi et al. and van Lindert et al. suggest that the major drawbacks of keyhole approaches applied to deep targets are limited surgical maneuverability due to the narrow bone opening, and difficulties in changing operative strategies when necessary. ${ }^{8,27}$

Our cadaveric study model was designed to evaluate the differences of temporal lobe retraction between the keyhole and the classic temporal approaches, using the same experimental setting. All data were comparative data evaluated in the same condition and after the temporal lobe was retracted until the same target area was fully visualized. Clearly our model does not take into account several techniques that can be employed to accrue space when using these approaches in clinical practice, such as CSF drainage (lumbar drain), hyperventilation, osmotic diuresis, and positioning. Our data were comparative data, generated by comparing the anatomical and surgical space volume in the same pair of approaches using cadaveric specimens. Each pair was executed on the same side and on the same cadaver, therefore minimizing variability as much as possible and maximizing the robustness of the data obtained in the 4 specimens. Our data show that there are no significant differences in temporal lobe retraction between the classic craniotomy and the keyhole craniotomy. A keyhole craniotomy does not diminish temporal lobe retraction. In addition, the retractor affected the temporal lobe not only at the site of its placement, but also along the anteroposterior horizontal direction. Even though the volume of the anatomical space is the same in both craniotomies, the surgical corridor of the keyhole craniotomy uses only a mean of $47 \%$ of this anatomical space whereas a classic craniotomy uses a mean of $87 \%$. While the temporal lobe is retracted, the temporal basal veins were stretched. The keyhole craniotomy is unable to visualize this stretching by using the microscope: it can only be seen, and then only to a limited extent, using the endoscope.

The section and displacement of the zygomatic arch as an adjunct to the pterional approach was first described in the 1980s by Fujitzu ${ }^{10}$ and Kuwabara. The authors suggested that detaching the zygomatic arch extensively exposes the anterior temporal base, allowing an oblique upward access to the interpeduncular fossa along the lowest possible supratentorial route, and this approach is useful to better access the interpeduncular cistern. $., 6,7,10,13,24$ Honeybul and colleagues suggest that the transzygomatic approach has minimal morbidity attributable to the access and has high-

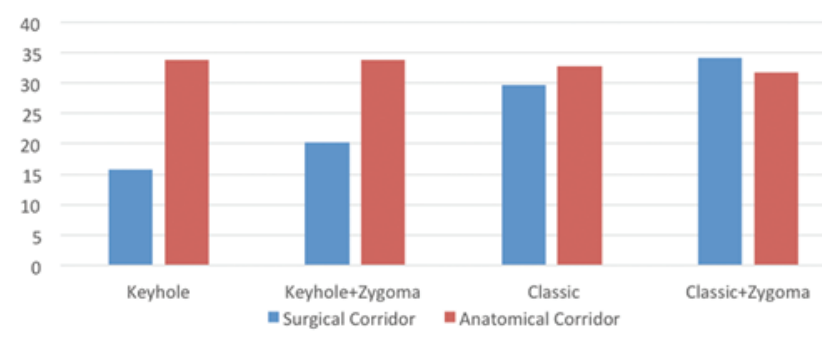

FIG. 6. Bar graph illustrating the difference between surgical and anatomical volume $\left(\mathrm{cm}^{3}\right)$. Figure is available in color online only. 


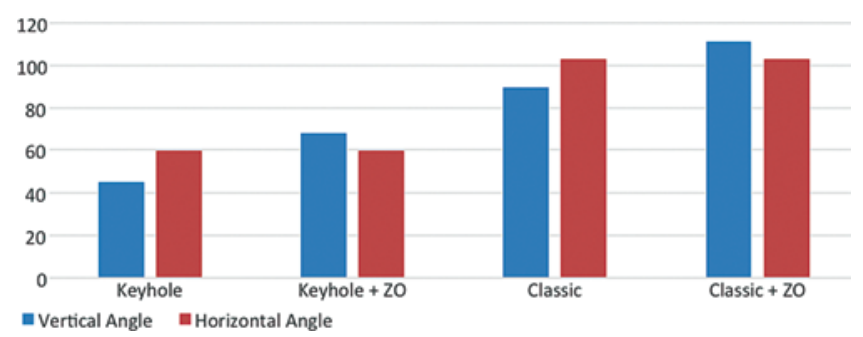

FIG. 7. Surgical maneuverability is determined by using vertical and horizontal angles $\left({ }^{\circ}\right)$; the bar graph illustrates the differences between the keyhole and classic approaches. ZO = zygomatic arch osteotomy. Figure is available in color online only.

lighted the versatility of the technique. ${ }^{12,13}$ Al-Mefty et al. proposed that it is necessary to section the zygomatic arch to gain access to the infratemporal fossa. ${ }^{1}$ According to Campero et al. and Melamed et al., the main advantage of the transzygomatic approach is that of providing major access to the floor of the middle fossa, without the obstacle of the temporal muscle. This is useful for advancing topographically in an extradural/intradural manner toward the floor of the middle fossa, with minimal retraction of the tip of the temporal lobe backward, and continuing in an inferior to superior direction to resect lesions located in the insular region. 4,20

Our results were similar to previous studies in that removal of the zygomatic arch enabled a more inferosuperior visual corridor to the target. Due to this inferior corridor, the retraction distance was reduced for both the keyhole and classic craniotomy at the lateral region $(17.11 \mathrm{~mm}$ and $16.43 \mathrm{~mm}$, respectively). However, medial retraction was not affected by zygomatic osteotomy. Clearly the advantages of the zygomatic osteotomy and of inferior temporalis retraction are downplayed in a cadaveric model due to the inevitable atrophy of the muscle in cadavers. This is due to the anatomical shape of the middle temporal fossa where the skull base and zygomatic arch are located under the level of the target. Thus, zygomatic arch removal advances the approach to the base of the temporal fossa, but it is not effective if the target sits deep and above the arch (Fig. 4).

Regarding the volume of the surgical corridor, zygomatic arch removal added $13 \%-17 \%$ volume to both the keyhole and the classic approaches. The benefit of this expanded volume is observed on surgical maneuvers because the larger surgical corridor enables more surgical attack angles. In accordance with previous studies, we determined that the fundamental advantage of the zygomatic arch extended subtemporal approach is that it provides excellent exposure and maneuverability to deep lesions due to the extra $44^{\circ}$ angles it provides to both keyhole and classic craniotomies. ${ }^{7}, 10$

\section{Limitations of the Study}

This is a cadaveric study with all the limitations of such a study (brain stiffness, lack of CSF, etc.). However, this is a comparative study, so its findings are robust in such an experimental setting. In addition, as minimally invasive approaches are proposed more and more often and as meaningful comparative clinical studies are lacking, perhaps relevant basic information may be provided by studies such as ours.

\section{Conclusions}

In our model, the keyhole subtemporal craniotomy was not associated with less temporal lobe retraction than the standard subtemporal approaches. While the anatomical space was the same in both craniotomies, the surgical space, the space in which one can execute surgical maneuvers, was larger in the standard subtemporal craniotomy than in the keyhole craniotomy. In addition, the standard craniotomy allowed visualization of the stretched temporal lobe basal vein so that maneuvers to control/limit this stretching may be undertaken. This venous visualization was limited in the keyhole approaches, where it was only achieved partially by using the endoscope. Zygomatic arch osteotomy facilitated the maneuverability and the surgical volume, but the temporal lobe retraction benefits were limited only to the lateral part of the temporal lobe with no effect on the deep temporal lobe retraction. The answer to our question "Is less always better?" is that perhaps less is not always better.

\section{References}

1. al-Mefty O, Anand VK: Zygomatic approach to skull-base lesions. J Neurosurg 73:668-673, 1990

2. Ansari SF, Terry C, Cohen-Gadol AA: Surgery for vestibular schwannomas: a systematic review of complications by approach. Neurosurg Focus 33(3):E14, 2012

3. Bernardo A, Evins AI, Tsiouris AJ, Stieg PE: A percutaneous transtubular middle fossa approach for intracanalicular tumors. World Neurosurg 84:132-146, 2015

4. Campero A, Campero AA, Socolovsky M, Martins C, Yasuda A, Basso A, et al: The transzygomatic approach. J Clin Neurosci 17:1428-1433, 2010

5. Cantore G, Delfini R, Ciappetta P: Surgical treatment of petroclival meningiomas: experience with 16 cases. Surg Neurol 42:105-111, 1994

6. Chotai S, Kshettry VR, Petrak A, Ammirati M: Lateral transzygomatic middle fossa approach and its extensions: surgical technique and 3D anatomy. Clin Neurol Neurosurg 130:33-41, 2015

7. Deda H, Ugur HC: Zygomatic anterior subtemporal approach for lesions in the interpeduncular cistern. Skull Base 11:257264, 2001

8. Fatemi N, Dusick JR, de Paiva Neto MA, Malkasian D, Kelly DF: Endonasal versus supraorbital keyhole removal of craniopharyngiomas and tuberculum sellae meningiomas. Neurosurgery 64 (5 Suppl 2):269-286, 2009

9. Fischer G, Stadie A, Reisch R, Hopf NJ, Fries G, BöcherSchwarz H, et al: The keyhole concept in aneurysm surgery: results of the past 20 years. Neurosurgery 68 (1 Suppl Operative):45-51, 2011

10. Fujitsu K, Takeo K: Zygomatic anterior subtemporal approach for lesions in the interpeduncular cistern. J Neurosurg 62:340-343, 1985

11. Ginzkey C, Scheich M, Harnisch W, Bonn V, EhrmannMüller D, Shehata-Dieler W, et al: Outcome on hearing and facial nerve function in microsurgical treatment of small vestibular schwannoma via the middle cranial fossa approach. Eur Arch Otorhinolaryngol 270:1209-1216, 2013

12. Honeybul S, Neil-Dwyer G, Evans BT, Lang DA: The tran- 
szygomatic approach: an anatomical study. Br J Oral Maxillofac Surg 35:334-340, 1997

13. Honeybul S, Neil-Dwyer G, Lang DA, Evans BT, Lees PD: The transzygomatic approach: a long-term clinical review. Acta Neurochir (Wien) 136:111-116, 1995

14. Jittapiromsak P, Wu A, Nakaji P, Spetzler RF, Preul MC: The challenge of access to the pontomesencephalic junction: an anatomical study of lateral approach and exposure. Skull Base 20:311-320, 2010

15. Kawase T: Endoscopic middle fossa approach for central skull base. World Neurosurg 80:505-506, 2013

16. Kawase T: Will endoscopic surgery be a messiah for midline skull base? World Neurosurg 81:273-274, 2014

17. Kim YD, Mendes GAC, Seoane P, Agrawal A, Maramreddy N, Nakaji P, et al: Quantitative anatomical study of tailored far-lateral approach for the VA-PICA regions. J Neurol Surg B Skull Base 76:57-65, 2015

18. Komatsu F, Komatsu M, Di Ieva A, Tschabitscher M: Endoscopic extradural subtemporal approach to lateral and central skull base: a cadaveric study. World Neurosurg 80:591-597, 2013

19. Li Z, Lan Q: Retrosigmoid keyhole approach to the posterior cranial fossa: an anatomical and clinical study. Eur Surg Res 44:56-63, 2010

20. Melamed I, Tubbs RS, Payner TD, Cohen-Gadol AA: Transzygomatic middle cranial fossa approach to access lesions around the cavernous sinus and anterior parahippocampus: a minimally invasive skull base approach. Acta Neurochir (Wien) 151:977-982, 2009

21. Reisch R, Perneczky A, Filippi R: Surgical technique of the supraorbital key-hole craniotomy. Surg Neurol 59:223-227, 2003

22. Salma A, Alkandari A, Sammet S, Ammirati M: A new methodology for laboratory evaluation of neurosurgical approaches based on the volume and shape of the surgical space with a mathematical model to quantify the surgical maneuverability in laboratory settings. J Neurol Surg B Skull Base 73:64-70, 2012

23. Salma A, Makiese O, Reiss A, Pillai P, Sammet S, Ammirati $\mathrm{M}$ : A microanatomical map of the structures hidden in the middle fossa based on the facial nerve hiatus: measurements and their variability. Clin Anat 26:436-443, 2013

24. Sindou M, Emery E, Acevedo G, Ben-David U: Respective indications for orbital rim, zygomatic arch and orbito-zygomatic osteotomies in the surgical approach to central skull base lesions. Critical, retrospective review in 146 cases. Acta Neurochir (Wien) 143:967-975, 2001

25. Sughrue ME: Minimally invasive approaches to the pineal region. Neurosurg Clin N Am 22:381-384, viii, 2011
26. Taniguchi M, Perneczky A: Subtemporal keyhole approach to the suprasellar and petroclival region: microanatomic considerations and clinical application. Neurosurgery 41:592-601, 1997

27. van Lindert E, Perneczky A, Fries G, Pierangeli E: The supraorbital keyhole approach to supratentorial aneurysms: concept and technique. Surg Neurol 49:481-490, 1998

28. Wang H, Zhang R, Yu W, Zhong P, Tan D: The posterior subtemporal keyhole approach combined with the transchoroidal approach to the ambient cistern: microsurgical anatomy and image-guided quantitative analysis. Acta Neurochir (Wien) 152:1933-1942, 2010

29. Wang H, Zhou F, Zhang R, Zhong P, Tan D: Opening cranial cisterns by the anterior subtemporal keyhole approach to the superior petroclival region: anatomical study and comparative analysis. Surg Neurol 72:124-130, 2009

30. Whitfield PC, Hardy DG: Cerebello-pontine angle tumors, in Moore AJ, Newell DW (eds): Neurosurgery: Principles and Practice. London: Springer, 2005, pp 247-262

31. Zhu W, Mao Y, Zhou LF, Zhang R, Chen L: Combined subtemporal and retrosigmoid keyhole approach for extensive petroclival meningioma surgery: report of experience with 7 cases. Minim Invasive Neurosurg 50:106-110, 2007

32. Zhu W, Mao Y, Zhou LF, Zhang R, Chen L: Combined subtemporal and retrosigmoid keyhole approach for extensive petroclival meningiomas surgery: report of experience with 7 cases. Minim Invasive Neurosurg 51:95-99, 2008

\section{Disclosures}

The authors report no conflict of interest concerning the materials or methods used in this study or the findings specified in this paper.

\section{Author Contributions}

Conception and design: Ammirati, Ercan. Acquisition of data: Ammirati, Ercan, Scerrati, Zhang. Analysis and interpretation of data: Ammirati, Ercan, Wu. Drafting the article: Ercan, Wu. Critically revising the article: Ammirati, Ercan. Statistical analysis: Ercan.

\section{Correspondence}

Mario Ammirati, Ohio State University Medical Center, Department of Neurological Surgery, 410 West 10th Ave., N1025 Doan Hall, Columbus, OH 43210. email: mario.ammirati@osumc.edu. 